\title{
UCRL-CONF-210535
}

LAW RENCE LIVERMORE N A T IO N A L LABORATORY

\section{Sequential detection of a weak target in a hostile ocean environment}

J. V. Candy, E. J. Sullivan

March 15, 2005

IEEE Conference Oceans 05 Europe Brest, France

June 20, 2005 through June 23, 2005 
This document was prepared as an account of work sponsored by an agency of the United States Government. Neither the United States Government nor the University of California nor any of their employees, makes any warranty, express or implied, or assumes any legal liability or responsibility for the accuracy, completeness, or usefulness of any information, apparatus, product, or process disclosed, or represents that its use would not infringe privately owned rights. Reference herein to any specific commercial product, process, or service by trade name, trademark, manufacturer, or otherwise, does not necessarily constitute or imply its endorsement, recommendation, or favoring by the United States Government or the University of California. The views and opinions of authors expressed herein do not necessarily state or reflect those of the United States Government or the University of California, and shall not be used for advertising or product endorsement purposes. 


\section{Sequential detection of a weak target in a hostile ocean environment}

IEEE CONFERENCE OCEANS'05 EUROPE

\author{
James V. Candy \\ Lawrence Livermore National Lab \\ P.O. Box 808, L-156 \\ Livermore, CA 94551 \\ (925) 422-8675 \\ candy1@1lnl.gov
}

\author{
Edmund J. Sullivan \\ E. J. S. Consultants \\ 846 Lawton Brook Lane \\ Portsmouth, RI 02871 \\ (401) 849-0356 \\ paddypriest@aol.com
}

\begin{abstract}
When the underlying physical phenomenology (medium, sediment, bottom, etc.) is space-time varying along with corresponding nonstationary statistics characterizing noise and uncertainties, then sequential methods must be applied to capture the underlying processes. Sequential detection and estimation techniques offer distinct advantages over batch methods. A reasonable signal processing approach to solve this class of problem is to employ adaptive or parametrically adaptive signal models and noise to capture these phenomena. In this paper, we develop a sequential approach to solve the signal detection problem in a nonstationary environment.
\end{abstract}

\section{INTRODUCTION}

The advent of quiet nuclear submarines and the current proliferation of even quieter diesel powered vessels have necessitated the need for more sophisticated signal processing techniques for target detection. Improvements in processor design can be achieved by developing approaches that incorporate knowledge of the surrounding ocean environment and noise in the form of mathematical models into their processing schemes [1-4]. One of the major problems with model-based signal processing schemes is assuring that the model incorporated in the algorithm is adequate for the proposed application and that it can faithfully represent the on-going phenomenology. Therefore, it is necessary, as part of the model-based processor (MBP) design procedure, to estimate/update the model parameters jointly (adaptively) while performing the required processing $[5,6]$. The incorporation of a parametrically adaptive, on-line, MBP can offer a dramatic detection improvement in a tactical passive or active sonar-type system especially when a rapid environmental assessment and detection is required. In this paper, we discuss the development of a processor capable of adapting to the ever-changing ocean environment thereby providing the required signal enhancement for detection.

With this motivation in mind, we investigate the development of a sequential detector, that is, a monitor that incorporates an initial mathematical representation of the weak target model into its framework and adapts, on-line, its parameters as the ocean and target change. In this paper we use an adaptive state-space scheme [7] and apply it to the detection and monitoring problem. This can be accomplished by constructing an adaptive MBP that allows continuous updating of the model parameters and is easily implemented by augmenting them into the current state vector [7-9]. Currently, techniques that adjust model parameters to adapt to the changing environment are termed environmentally adaptive.

We start by developing a solution to the general sequential detection problem. Here we assume generic signal models, uncertainty and noise. It is shown that the solution can be obtained using the Wald sequential theoretic framework leading to a recursive log-likelihood solution under the Neyman-Pearson criterion [10]. Once the basic detector is developed, structurally, we extend the results to solve the composite detection problem with unknown parameters leading to the generalized (sequential) log-likelihood solution. Here we incorporate both the log-likelihood decision function and adaptive parameter estimation into the sequential framework leading to a joint detection/signal enhancement solution.

Special cases of the generic signal model are then investigated (e.g. far-field sources, unknown frequencies, bearings, etc.) and cast into the sequential framework to develop the underlying theory required for each. It is shown that this formulation of the detection problem for nonstationary ocean acoustic signals leads to a general approach capable of solving a large number of detection/enhancement problems.

\section{SEQUENTIAL DETECTION THOERY}

In this section we develop the sequential detection scheme for nonstationary processes. We start with the batch solution and then develop the sequential approach. First we assume that we have a set of vector measurements defined by $\mathrm{P}_{\mathrm{t}}:=\{\mathbf{p}(0), \mathbf{p}(1), \cdots, \mathbf{p}(t)\}, \mathbf{p} \in \mathbb{C}^{L \times 1}$ and we would like to 
develop a processor based on the usual binary decision problem [10]:

$$
\begin{aligned}
& \mathrm{H}_{\mathrm{o}}: \mathbf{p}(t)=\mathbf{v}(t) \\
& \mathrm{H}_{1}: \mathbf{p}(t)=\mathbf{s}(t)+\mathbf{v}(t)
\end{aligned}
$$

where the null hypothesis is noise and the alternate is the signal and noise case. We choose the Neyman-Pearson criterion to develop our detector [10].

Recall that the Neyman-Pearson theorem states that a detector is optimal if it maximizes the probability of detection, $\operatorname{Pr}_{\mathrm{DET}}$ (or minimizes the miss probability) for any false alarm rate less than a pre-specified value, say $\operatorname{Pr}_{\mathrm{FA}}^{*}$. The theorem follows directly from a constrained optimization problem formulation using Lagrange multipliers (see [10,11] for details), which yields the solution based on the ratio of likelihood's, $\operatorname{Pr}\left(\mathrm{P}_{t} \mid H_{i}\right) ; i=0,1$ (for the binary case). That is, to maximize $\operatorname{Pr}_{\mathrm{DET}}$ for a fixed value of $\operatorname{Pr}_{\mathrm{FA}}=\operatorname{Pr}_{\mathrm{FA}}^{*}$, we have the likelihood ratio, $L(t)$ or equivalently sufficient statistic defined by the joint density functions

$$
L(t):=L\left(\mathrm{P}_{t}\right)=\frac{\operatorname{Pr}\left(\mathrm{P}_{t} \mid H_{1}\right)}{\operatorname{Pr}\left(\mathrm{P}_{t} \mid H_{o}\right)} \quad>^{\text {Accept } H_{1}} \mathrm{~T},
$$

for $\mathrm{P}_{t}$ and $\operatorname{Pr}(\cdot)$ are the respective joint probabilities under each hypothesis. Here the threshold $\mathrm{T}$ is found from the false alarm density for the pre-specified value

$$
\operatorname{Pr}_{\mathrm{FA}}=\int_{\mathrm{T}}^{\infty} \operatorname{Pr}\left(\mathrm{P}_{t} \mid H_{o}\right) d P=\operatorname{Pr}_{\mathrm{FA}}^{*} .
$$

Thus, based on the Neyman-Pearson criterion, the optimal detector can be found for "batch" mode as

$$
L(t)=\frac{\operatorname{Pr}\left(\mathrm{P}_{t} \mid H_{1}\right)}{\operatorname{Pr}\left(\mathrm{P}_{t} \mid H_{o}\right)}=\frac{\operatorname{Pr}\left(\mathbf{p}(t), \cdots, \mathbf{p}(0) \mid H_{1}\right)}{\operatorname{Pr}\left(\mathbf{p}(t), \cdots, \mathbf{p}(0) \mid H_{o}\right)} .
$$

It follows from the chain rule of probability theory [10] that

$$
\begin{aligned}
\operatorname{Pr}\left(\mathrm{P}_{t} \mid H_{i}\right)= & \prod_{k=0}^{t} \operatorname{Pr}\left(\mathbf{p}(t-k) \mid \mathrm{P}_{t-k-1} ; H_{i}\right) \\
= & \operatorname{Pr}\left(\mathbf{p}(t) \mid \mathrm{P}_{t-1} ; H_{i}\right) \times \operatorname{Pr}\left(\mathbf{p}(t-1) \mid \mathrm{P}_{t-2} ; H_{i}\right) \times \cdots \times \\
& \operatorname{Pr}\left(\mathbf{p}(1) \mid \mathbf{p}(0) ; H_{i}\right) \times \operatorname{Pr}\left(\mathbf{p}(0) ; H_{i}\right)
\end{aligned}
$$

which can be expressed succinctly using Bayes' rule as

$$
\operatorname{Pr}\left(\mathrm{P}_{t} \mid H_{i}\right)=\operatorname{Pr}\left(\mathbf{p}(t), \mathrm{P}_{t-1} \mid H_{i}\right)=\operatorname{Pr}\left(\mathbf{p}(t) \mid \mathrm{P}_{t-1} ; H_{i}\right) \operatorname{Pr}\left(\mathrm{P}_{t-1} \mid H_{i}\right)
$$

Substituting these expressions into Eq. (2), we obtain
$L(t)=\left[\frac{\operatorname{Pr}\left(\mathrm{P}_{t-1} \mid H_{1}\right)}{\operatorname{Pr}\left(\mathrm{P}_{t-1} \mid H_{o}\right)}\right] \frac{\operatorname{Pr}\left(\mathbf{p}(t) \mid \mathrm{P}_{t-1} ; H_{1}\right)}{\operatorname{Pr}\left(\mathbf{p}(t) \mid \mathrm{P}_{t-1} ; H_{o}\right)}=L(t-1) \frac{\operatorname{Pr}\left(\mathbf{p}(t) \mid \mathrm{P}_{t-1} ; H_{1}\right)}{\operatorname{Pr}\left(\mathbf{p}(t) \mid \mathrm{P}_{t-1} ; H_{o}\right)}$

which is precisely the sequential form of the likelihood ratio. It is also clear that not just the likelihood function can be used but any monotonic function of the likelihood can also be used as well $[10,11]$. Taking natural logarithms of both sides of the equation, and defining $\Lambda(t):=\ln L(t)$, we obtain the sequential log-likelihood ratio as

$\Lambda(t)=\Lambda(t-1)+\ln \operatorname{Pr}\left(\mathbf{p}(t) \mid \mathrm{P}_{t-1} ; H_{1}\right)-\ln \operatorname{Pr}\left(\mathbf{p}(t) \mid \mathrm{P}_{t-1} ; H_{o}\right)$.

To construct the sequential probability ratio test (SPRT) or equivalently the sequential likelihood ratio test (SLRT) based on the Neyman-Pearson criterion, we must define two timevarying thresholds $\mathrm{T}_{o}(t)$ and $\mathrm{T}_{1}(t)$. If the likelihood ratio at time $t$ is greater than threshold $\mathrm{T}_{1}(t)$, we accept hypothesis $H_{1}$. If it is less than $\mathrm{T}_{o}(t)$, we accept $H_{o}$, but if its value lies between the thresholds, we continue to take another sample. The sequential test differs from the fixed sample size "batch" test and is capable of handling nonstationary statistics as we will show in a subsequent section.

To implement the SLRT at the $t^{\text {th }}$ stage ( see Eq. (7) or (8)), we must know the prior probabilities or equivalently the prior likelihood ratio, $L(t-1)$ (or $\Lambda(t-1)$ ) containing all of the past information and the corresponding stage conditional probabilities $\operatorname{Pr}\left(\mathbf{p}(t) \mid \mathrm{P}_{t-1} ; H_{i}\right)$ to sequentially update the likelihood. The $t^{\text {th }}$ stage thresholds are construct following Neyman-Pearson theory in terms of the detection and false alarm probabilities as

$\mathrm{T}_{o}(t)=\frac{\operatorname{Pr}_{\mathrm{DET}}}{\mathrm{Pr}_{\mathrm{FA}}}=\frac{1-\mathrm{Pr}_{\mathrm{MISS}}}{\operatorname{Pr}_{\mathrm{FA}}}$, and $\mathrm{T}_{1}(t)=\frac{1-\mathrm{Pr}_{\mathrm{DET}}}{1-\mathrm{Pr}_{\mathrm{FA}}}=\frac{\operatorname{Pr}_{\mathrm{MISS}}}{1-\operatorname{Pr}_{\mathrm{FA}}}$

In the case of the log-likelihood, the thresholds must be transformed as well, that is,

$$
\begin{aligned}
& \left.\Lambda(t) \geq \ln \mathrm{T}_{1}(t) \quad \text { [Accept } H_{1}\right] \\
& \ln _{\mathrm{o}}(t)<\Lambda(t)<\ln \mathrm{T}_{1}(t) \quad \text { [Continue] } \\
& \Lambda(t) \leq \ln \mathrm{T}_{\mathrm{o}}(t) \quad \text { [Accept } H_{o} \text { ] }
\end{aligned}
$$

This completes the fundamental concepts for the construction of the sequential detection approach; however, before we close this section, we must consider the case of incorporating unknown parameters into the binary detection problem, which will lead to the generalized likelihood tests [11].

If the conditional densities of the underlying problem contain 
a set of unknown parameters defined by the vector $\boldsymbol{\Theta}$, then two cases must be considered for sequential detection. In the first case, $\Theta$ is considered to be random and therefore joint conditional probabilities, $\operatorname{Pr}\left(\mathrm{P}_{t}, \boldsymbol{\Theta} \mid H_{i}\right)$ must be incorporated and the likelihood is now computed in terms of the marginals

$$
\begin{aligned}
L(t)=\frac{\operatorname{Pr}\left(\mathrm{P}_{t-1} \mid H_{1}\right)}{\operatorname{Pr}\left(\mathrm{P}_{t-1} \mid H_{o}\right)} & =\frac{\int \operatorname{Pr}\left(\mathrm{P}_{t-1}, \boldsymbol{\Theta} \mid H_{1}\right) d \Theta}{\int \operatorname{Pr}\left(\mathrm{P}_{t-1}, \boldsymbol{\Theta} \mid H_{o}\right) d \Theta} \\
& =\frac{\int \operatorname{Pr}\left(\mathrm{P}_{t-1} \mid \boldsymbol{\Theta} ; H_{1}\right) \operatorname{Pr}\left(\boldsymbol{\Theta} \mid H_{1}\right) d \Theta}{\int \operatorname{Pr}\left(\mathrm{P}_{t-1} \mid \boldsymbol{\Theta} ; H_{o}\right) \operatorname{Pr}\left(\boldsymbol{\Theta} \mid H_{o}\right) d \Theta}
\end{aligned}
$$

by integrating over $\boldsymbol{\Theta}$, the likelihood ratio is determined and in a sense, the composite problem is reduced to simple hypothesis testing.

In the second case, $\boldsymbol{\Theta}$ is considered to be deterministic but unknown and the above approach cannot be applied. Another approach is to estimate the unknown parameter vector $\hat{\boldsymbol{\Theta}} \rightarrow \boldsymbol{\Theta}$ under each hypothesis and proceed with the simple testing. A maximum likelihood estimate, $\hat{\boldsymbol{\Theta}}_{\mathrm{ML}}$, can be used to create the generalized likelihood ratio test (GLRT) defined by

$$
L_{\boldsymbol{\Theta}}(t):=L_{\boldsymbol{\Theta}}\left(\mathrm{P}_{t}\right)=\frac{\max _{\Theta_{1}} \operatorname{Pr}\left(\mathrm{P}_{t} \mid \boldsymbol{\Theta}_{1} ; H_{1}\right)}{\max _{\Theta_{o}} \operatorname{Pr}\left(\mathrm{P}_{t} \mid \mathbf{\Theta}_{o} ; H_{o}\right)}
$$

This is the approach we use in this paper for our adaptive processor; therefore, the GLRT is defined by

$$
L_{\boldsymbol{\Theta}}(t)=\frac{\operatorname{Pr}\left(\mathrm{P}_{t} \mid \boldsymbol{\Theta}_{1}, H_{1}\right)}{\operatorname{Pr}\left(\mathrm{P}_{t} \mid \boldsymbol{\Theta}_{o}, H_{o}\right)}=L_{\boldsymbol{\Theta}}(t-1) \frac{\operatorname{Pr}\left(\mathbf{p}(t) \mid \mathrm{P}_{t-1 ;} ; \boldsymbol{\Theta}_{1}, H_{1}\right)}{\operatorname{Pr}\left(\mathbf{p}(t) \mid \mathrm{P}_{t-1 ;} \boldsymbol{\Theta}_{o}, H_{o}\right)}
$$

or in logarithmic form as

$$
\Lambda_{\boldsymbol{\Theta}}(t)=\Lambda_{\boldsymbol{\Theta}}(t-1)+\ln \operatorname{Pr}\left(\mathbf{p}(t) \mid \mathrm{P}_{t-1} ; \boldsymbol{\Theta}_{1}, H_{1}\right)-\ln \operatorname{Pr}\left(\mathbf{p}(t) \mid \mathrm{P}_{t-1} ; \boldsymbol{\Theta}_{o}, H_{o}\right)
$$

with the corresponding thresholds in terms of the parameters

$$
\begin{aligned}
& \mathrm{T}_{o}(t ; \boldsymbol{\Theta}):=\frac{1-\operatorname{Pr}_{\mathrm{MISS}}(\boldsymbol{\Theta})}{\operatorname{Pr}_{\mathrm{FA}}(\boldsymbol{\Theta})}, \text { and } \mathrm{T}_{1}(t ; \boldsymbol{\Theta}):=\frac{\operatorname{Pr}_{\mathrm{MISS}}(\boldsymbol{\Theta})}{1-\operatorname{Pr}_{\mathrm{FA}}(\boldsymbol{\Theta})} \\
& \text { for } \\
& \operatorname{Pr}_{\mathrm{FA}}(\boldsymbol{\Theta})=\int_{\mathrm{T}_{o}}^{\infty} \operatorname{Pr}\left(\mathrm{P}_{t} \mid \boldsymbol{\Theta}_{o}, H_{o}\right) d P ; \operatorname{Pr}_{\mathrm{MISS}}(\boldsymbol{\Theta})=\int_{\mathrm{T}_{o}}^{\infty} \operatorname{Pr}\left(\mathrm{P}_{t} \mid \boldsymbol{\Theta}_{1}, H_{1}\right) d P
\end{aligned}
$$

This completes the background theory, next we apply it to our ocean acoustic problem.

\section{JOINT SEQUENTIAL DETECTION/SIGNAL ENHANCEMENT}

In this section we develop a solution to the joint detection /signal enhancement problem for a towed array in the ocean. We start with the noisy pressure-field measurement given by

$$
p\left(r_{\ell} ; t\right)=s\left(r_{\ell} ; t\right)+v\left(r_{\ell} ; t\right)
$$

where $p$ is the measured pressure-field at the $\ell^{\text {th }}$-hydrophone located at spatial location, $r_{\ell}$, and at time $t ; s$ is the target or source signal to be detected and $v$ is the broadband noise component present in the ocean environment. We can simplify this notation by expanding over the horizontal array of $L$-elements, that is,

$$
\mathbf{p}(t)=\mathbf{s}(t)+\mathbf{v}(t)
$$

with $\mathbf{p}, \mathbf{s}, \mathbf{v} \in \mathbb{C}^{L \times 1}$. We decompose this representation further by developing the component signal and noise models. We assume that the signal can be characterized by a weak target in the far-field of the array given by

$$
s(t)=\alpha_{o} e^{i\left(\omega_{o} t-\mathbf{k}_{o} \cdot \mathbf{r}\right)}=\alpha_{o} e^{i\left(\omega_{o} t-k_{o} \sin \theta_{o}\left(r_{o}+v t\right)\right)},
$$

for the target parameters: $\alpha_{o}, \omega_{o}, k_{o}, \theta_{o}, r_{o}$ that are the respective amplitude, temporal frequency, wavenumber, bearing angle and initial sensor location. Since the array is being towed, we include the tow speed, $v$ as well. We can simplify this model by defining the following terms,

$$
s(t)=\alpha_{o}(t) e^{-i \beta_{o}(t) \sin \theta_{o}},
$$

for $\alpha_{o}(t):=\alpha_{o} e^{i \omega_{o} t}$ and $\beta_{\mathrm{o}}(t):=k_{o}\left(r_{o}+v t\right)$. The broadband measurement noise is modeled as zero-mean, white gaussian, $\mathbf{v} \sim \mathrm{N}\left(0, R_{v v}(t)\right)$. Note that we are not restricting the statistics to be stationary, so we can accommodate the nonstationarities (transients, etc.) that occur naturally in the ocean environment.

For the joint detection/signal enhancement problem under the null hypothesis, we have that $\operatorname{Pr}\left(\mathbf{p}(t) \mid \mathrm{P}_{\mathrm{t}-1} ; H_{o}\right)$ is a conditionally gaussian distribution, since $\mathbf{v}$ is white. Therefore the conditional density is given by $\operatorname{Pr}\left(\mathbf{p}(t) \mid \mathrm{P}_{t-1} ; H_{o}\right) \sim \mathrm{N}\left(0, R_{v v}(t)\right)$. For various assumptions on the structure of the signal model the density changes. We start with the simplest signal model and proceed.

Case I: Known signal $\mathbf{s}(t)$ with $\operatorname{Pr}\left(\mathbf{p}(t) \mid \mathrm{P}_{t-1} ; H_{1}\right) \sim \mathrm{N}\left(\hat{\mathbf{p}}(t \mid t-1), R_{e_{p} \rho_{p}}(t)\right)$ : For this case we have the following conditional statistics 


$$
\begin{aligned}
& \hat{\mathbf{p}}(t \mid t-1)=E\left\{\mathbf{s}(t)+\mathbf{v}(t) \mid \mathrm{P}_{t-1}\right\}=\mathbf{s}(t) \\
& \mathbf{e}_{\mathrm{p}}(t)=\mathbf{p}(t)-\hat{\mathbf{p}}(t \mid t-1)=\mathbf{s}(t)+\mathbf{v}(t)-\mathbf{s}(t)=\mathbf{v}(t) \\
& R_{\mathbf{e}_{\mathrm{p}} \mathbf{e}_{\mathrm{p}}}(t)=R_{\mathrm{vv}}(t)
\end{aligned}
$$

and the corresponding log-likelihood is therefore

$$
\begin{gathered}
\Lambda(t)=\Lambda(t-1)+\Delta K+\frac{1}{2}(\mathbf{p}(t)-\mathbf{s}(t))^{\prime} R_{v v}^{-1}(t)(\mathbf{p}(t)-\mathbf{s}(t)) \\
-\frac{1}{2} \mathbf{p}^{\prime}(t) R_{v v}^{-1}(t) \mathbf{p}(t)
\end{gathered}
$$

Simplifying terms Eq. (21) becomes

$$
\Lambda(t)=\Lambda(t-1)+\Delta K+\mathbf{p}^{\prime}(t) R_{v v}^{-1}(t) \mathbf{s}(t)+\mathbf{s}^{\prime}(t) R_{e_{\mathrm{p}} e_{\mathrm{p}}}^{-1}(t) \mathbf{s}(t)(22)
$$

where $\Delta K:=K_{1}-K_{o}=\frac{1}{2}\left(\ln \left|R_{v v}(t)\right|-\ln \left|R_{e_{\mathrm{p}} e_{\mathrm{p}}}(t)\right|\right)$. Moving the terms that are not a function of the measurement into the threshold gives the classical matched-filter result $[10,11]$.

Case II:Unknown $\mathbf{s}(t ; \Theta)$ for $\operatorname{Pr}\left(\mathbf{p}(t ; \Theta) \mid \mathrm{P}_{t-1} ; \Theta, H_{1}\right) \sim \mathrm{N}\left(\hat{\mathbf{p}}(t \mid t-1 ; \Theta), R_{e_{\mathrm{p}} e_{\mathrm{p}}}(t ; \Theta)\right)$

In this case the signal structure is known from Eq. (18), but not the parameters. The conditional statistics are therefore

$$
\begin{aligned}
& \hat{\mathbf{p}}(t \mid t-1 ; \Theta)=E\left\{\mathbf{s}(t)+\mathbf{v}(t) \mid \mathrm{P}_{t-1} ; \Theta\right\}=\hat{\mathbf{s}}(t ; \Theta) \\
& \mathbf{e}_{\mathrm{p}}(t ; \Theta)=\mathbf{p}(t)-\hat{\mathbf{p}}(t \mid t-1 ; \Theta)=(\mathbf{s}(t)-\hat{\mathbf{s}}(t ; \Theta))+\mathbf{v}(t)=\tilde{\mathbf{s}}(t ; \Theta)+\mathbf{v}(t) \\
& R_{\mathbf{e}_{\mathrm{p}} \mathbf{e}_{\mathrm{p}}}(t ; \Theta)=R_{\tilde{\mathbf{s}} \tilde{\mathbf{s}}}(t ; \Theta)+R_{v v}(t)
\end{aligned}
$$

Since $\mathbf{s}(t)$ is assumed to be a far-field source, we have that at the $\ell^{\text {th }}$-sensor, $s_{\ell}(t)=\alpha_{\ell}(t) e^{-i \beta_{\ell}(t) \sin \theta}$. Now expanding over the $L$-sensor array, we obtain the signal vector

$$
\mathbf{s}(t)=\left[\begin{array}{c}
\alpha(t) e^{-i k \beta_{1}(t) \sin \theta} \\
\vdots \\
\alpha(t) e^{-i k \beta_{L}(t) \sin \theta}
\end{array}\right]=\left[\begin{array}{c}
\alpha e^{i \omega t} e^{-i k\left(x_{1}+v t\right) \sin \theta} \\
\vdots \\
\alpha e^{i \omega t} e^{-i k\left(x_{L}+v t\right) \sin \theta}
\end{array}\right]
$$

Signal enhancement can be achieved by defining the signal vector in terms of its unknown parameters, $\mathbf{s}(t ; \boldsymbol{\Theta})$, for (a single target), $\boldsymbol{\Theta}:=[\alpha|\omega| \theta]^{\prime}$. In this case we assume that the unknown parameters in the signal model, $\boldsymbol{\Theta}$, are characterized by a piecewise constant representation $(\dot{\boldsymbol{\Theta}}=\mathbf{0})$ This parameter vector is the state vector and therefore the underlying discrete Gauss-Markov model for this problem is

$$
\begin{aligned}
& \boldsymbol{\Theta}(t)=\boldsymbol{\Theta}(t-1)+\Delta t \mathbf{w}_{\Theta}(t-1) \\
& \mathbf{p}(t)=\mathbf{c}[t ; \boldsymbol{\Theta}]+\mathbf{v}(t)=\mathbf{s}(t ; \boldsymbol{\Theta})+\mathbf{v}(t)
\end{aligned}
$$

where $\mathbf{w}_{\Theta} \sim \mathrm{N}\left(0, R_{\mathrm{w}_{\Theta} \mathrm{w}_{\Theta}}\right)$. Thus, the basic detection/signal enhancement problem can now be stated as

GIVEN a set of discrete noisy pressure-field measurements, $\{\mathbf{p}(t)\}, t=1,2, \cdots, N_{t}$ and the GaussMarkov model of Eq. (25), FIND the optimal (NeymanPearson) solution for joint detection/enhancement. That is, find the log-likelihood $\Lambda_{\Theta}(t)$ and the best (minimum variance) estimate of the signal, $\hat{\mathbf{s}}(t ; \Theta)$ or equivalently the parameter vector $\hat{\boldsymbol{\Theta}}(t \mid t)$.

Using the GLRT of Eq. (12) to sequentially perform the detection, we must estimate the parameters on-line as well. It is well known from recursive estimation theory that a MBP can be used to sequentially estimate these parameters based on this Gauss-Markov formulation. We have a linear statespace, but unfortunately a nonlinear measurement system requiring a nonlinear processor. This problem can be solved by a parametrically adaptive MBP using the recursive extended Kalman filter (EKF) given in Table I to provide the maximum likelihood parameter estimates (gaussian case) for the optimal detector $[5,6,9]$.

TABLE I

ADAPTIVE MODEL-BASED SIGNAL ENHANCER

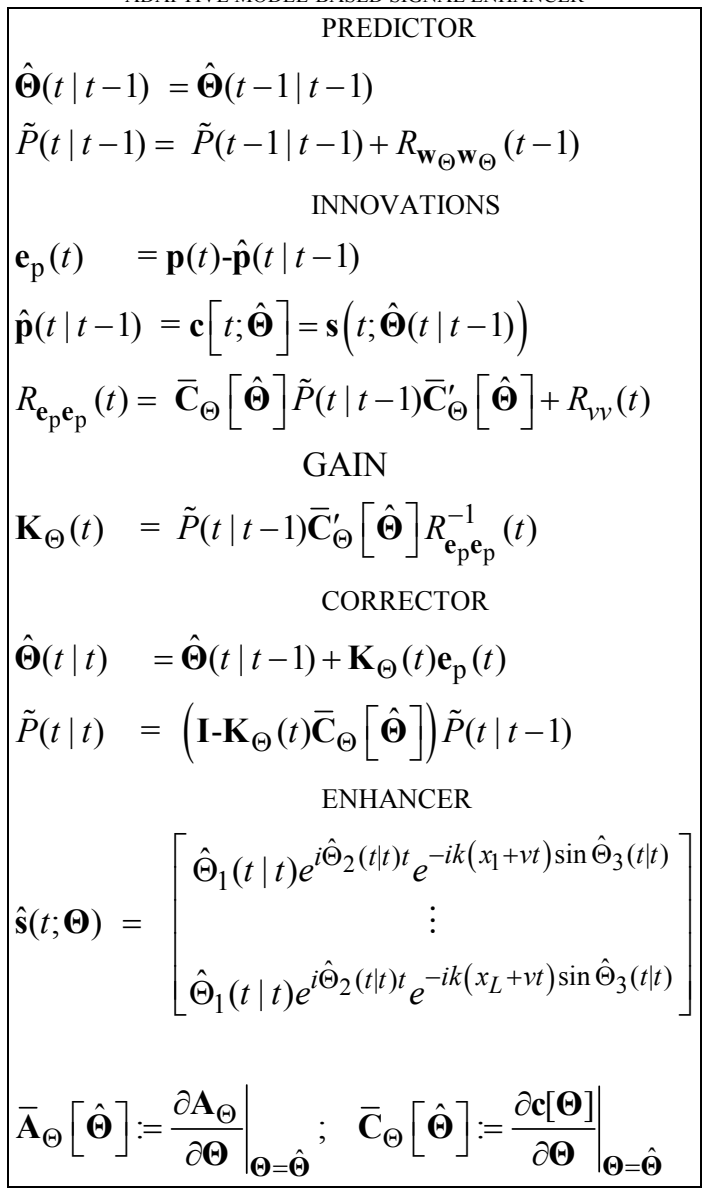


Using the EKF algorithm it is necessary to provide thejacobians for implementation, that is,

$$
\begin{gathered}
\frac{\partial A_{\Theta}[\boldsymbol{\Theta}]}{\partial \boldsymbol{\Theta}}=\mathrm{I}, \frac{\partial \mathrm{c}[\boldsymbol{\Theta}]}{\partial \theta}=i \alpha(t) \beta_{\ell}(t) \cos \theta e^{i \beta_{\ell}(t) \sin \theta} \\
\frac{\partial \mathrm{c}[\boldsymbol{\Theta}]}{\partial \omega}=i t \alpha(t) e^{i \beta_{\ell}(t) \sin \theta}, \frac{\partial \mathrm{c}[\boldsymbol{\Theta}]}{\partial \alpha}=e^{i\left(\omega t-\beta_{\ell}(t) \sin \theta\right)} \\
\ell=1, \cdots, L
\end{gathered}
$$

completing the development of the parametrically adaptive solution.

Using the same approach as Case I, we obtain the optimal decision function

$$
\Lambda_{\Theta}(t)=\Lambda_{\Theta}(t-1)+\Delta K+\mathbf{e}_{\mathbf{p}}^{\prime}(t ; \boldsymbol{\Theta}) R_{e_{\mathrm{p}} e_{\mathrm{p}}}^{-1}(t ; \boldsymbol{\Theta}) \mathbf{e}_{\mathbf{p}}(t ; \boldsymbol{\Theta})-\mathbf{p}^{\prime}(t) R_{v v}^{-1}(t) \mathbf{p}(t)
$$

The innovations and its covariance are given by the following (see Table I for details)

$$
\begin{array}{ll}
\mathbf{e}_{\mathrm{p}}(t ; \boldsymbol{\Theta}) & =\mathbf{p}(t)-\hat{\mathbf{p}}(t \mid t-1) \\
\hat{\mathbf{p}}(t \mid t-1 ; \boldsymbol{\Theta}) & =\mathbf{c}[t ; \hat{\boldsymbol{\Theta}}]=\mathbf{s}(t ; \hat{\boldsymbol{\Theta}}(t \mid t-1)) \\
R_{\mathbf{e}_{\mathrm{p}} \mathbf{e}_{\mathrm{p}}}(t ; \boldsymbol{\Theta}) & =\overline{\mathbf{C}}_{\Theta}[\hat{\boldsymbol{\Theta}}] \tilde{P}(t \mid t-1) \overline{\mathbf{C}}_{\Theta}^{\prime}[\hat{\boldsymbol{\Theta}}]+R_{v v}(t)
\end{array}
$$

So we see that the effect of the unknown signal model is to complicate the log-likelihood decision function by including the additional information. However, assuming that this knowledge is accurate, the correct decision can be accomplished at a much lower SNR---which is the main objective of this MBP scheme.

Thus, the joint parametrically adaptive processor is capable of not only providing a the optimal detection solution $\left(\Lambda_{\Theta}(t)\right)$ in a sequential manner with nonstationary statistics incorporated, but also capable of adaptively estimating the weak far-field target signal for optimal signal enhancement $(\hat{\mathbf{s}}(t ; \boldsymbol{\Theta}) \rightarrow \mathbf{s}(t))$. Thus, the sequential approach does in fact provide a solution to the joint detection/enhancement problem. Next we summarize our results and discuss future efforts.

\section{SUMMARY}

In this paper we have developed a solution to the joint detection/signal enhancement problem using a model-based approach [5]. Starting with the optimal (Neyman-Pearson) detector solution, we developed the corresponding modelbased solution demonstrating their equivalence for the case where the signal is known a priori. Next we developed the solution to the joint problem with the signal unknown, but parameterized as a far-field source. The solution to this problem lead to the parametrically adaptive model-based processor implemented with the nonlinear extended Kalman filter (EKF) algorithm. It was shown how to design the processor for this problem.

Future efforts will be aimed at applying this technique to both simulated and measured hydrophone data. We plan to use the discrete implementation of the factorized EKF available in MATLAB [12] with the toolbox SSPACK_PC [13].

\section{REFERENCES}

[1] C. S. Clay and H. Medwin, Acoustical Oceanography, John Wiley, New York, pp. 110-134, 1977.

[2] A. Tolstoy, Matched-Field Processing for Ocean Acoustics, World Scientific Pub. Co., 1993.

[3] A. B. Baggeroer, W. A. Kuperman and H. Schmidt. "Matched-filed processing: source localization in correlated noise as an optimum parameter estimation problem," Proc. IEEE, Vol. 69, (11), pp. 1451-1506, 1981.

[4] R. O. Nielsen, Sonar Signal Processing, Artech House, Boston, MA pp.95-140, 1991.

[5] J. V. Candy, Model-Based Signal Processing, John Wiley, New York, 2005.

[6] A. Jazwinski, Stochastic Processes and Filtering Theory, Academic Press, New York, 1970.

[7] J. V. Candy and E. J. Sullivan, "Model-based identification: an adaptive approach to ocean acoustic processing," IEEE J. Oceanic Engr., 21, (3) pp. 273289, 1996.

[8] J. V. Candy and E. J. Sullivan, "Passive localization in ocean acoustics: a model-based approach," $J$. Acoust. Soc. Am., 98 (3), pp. 1455-1471, 1995.

[9] E. J. Sullivan and J. V. Candy, "Space-time array processing: the model-based approach," J. Acoust. Soc. Am., Vol. 102 (5), Pt. 1, pp. 2809-2820, 1997.

[10] A. P. Sage and J. L. Melsa, Estimation Theory with Applications to Communications and Control. McGraw-Hill, New York, 1971.

[11] S. M. Kay, Fundamentals of Statistical Signal Processing: Detection Theory. Prentice-Hall, New Jersey, 1993.

[12] Mathworks, MATLAB User's Manual, Mathworks Inc., Boston, MA, 1993.

[13] J. V. Candy and P. M. Candy, "SSPACK_PC: A model-based signal processing package on personal computers," DSP Applications, Vol. 2 (3), 33-42, 1993.

This work was performed under the auspices of the U.S. Department of Energy by University of California, Lawrence Livermore National Laboratory under contract No. W-7405Eng-48. 